\title{
Impacts of Strongly Magnetized Degenerate Plasma on the Electron-capture Rates
}

\author{
Yudong Luo ${ }^{1,2, *}$, Motohiko Kusakabe ${ }^{3, * *}$, and Toshitaka Kajino ${ }^{1,2,3, * * *}$ \\ ${ }^{1}$ National Astronomical Observatory of Japan, 2-21-1 Osawa, Mitaka, Tokyo 181-8588, Japan \\ ${ }^{2}$ Graduate School of Science, The University of Tokyo, 7-3-1 Hongo, Bunkyo-ku, Tokyo 113-0033, \\ Japan \\ ${ }^{3}$ School of Physics, International Research Center for Big-Bang Cosmology and Element Genesis, Bei- \\ hang University, 37, Xueyuan Rd., Haidian-qu, Beijing 100083 China
}

\begin{abstract}
The strongly magnetized degenerate astrophysical plasma is investigated. A relativistic Hartree self-consistent field method is applied to calculate the screening potential. A profile from a $15 \mathrm{M}_{\odot}$ core collapsing supernova (SN) progenitor is applied to evaluate the electron-capture rates of ${ }^{54} \mathrm{Fe}$. It is found that the screening potential at high field is enhanced compared with the previous study. If the field is high enough and only the lowest Landau level is allowed, two orders of magnitude reduction of the capture rates are found in the highdensity region. Such deviations of the electron capture rates are essential since the rates determine the neutron richness of the progenitor model as well as the iron core mass, which are crucial for MHD-Jet SNe explosion calculation.
\end{abstract}

\section{Introduction}

The weak interactions determine the nucleosynthetic path in the supernovae ( $\mathrm{SNe}$ ) and also its explosion models [1-4]. For the MHD-Jet SNe, it is believed that the collapsing and bouncing phases have relativistic degenerate electron gas in a strong magnetic field of the order of $10^{14-16} \mathrm{G}[5]$ in the inner region. The thermodynamics of the electrons (positrons) in such an environment is different from the field-free case and could further deviate the nuclear weak interactions significantly. Prompted by this fact, this work focuses on the microscopic effects of the relativistic, magnetized degenerate plasmas on the weak interactions. The general Hartree self-consistent field method of the relativistic degenerate electron gas $[6,7]$ is applied to calculate the strong screening potential in the background magnetic field. The impacts on the weak interaction rates from the highly magnetized degenerate plasma are also investigated. We apply a profile from the $15 \mathrm{M}_{\odot}$ progenitor model to calculate the electroncapture rates of iron group nuclei. The results are essential to determine the iron core mass before the explosion and the electron fraction $Y_{e}$ during SN's collapsing phase.

\footnotetext{
*e-mail: yudong.luo@nao.ac.jp

**e-mail: kusakabe@buaa.edu.cn

***e-mail: kajino@buaa.edu.cn
} 


\section{Impacts of Strongly Magnetized Degenerate Plasma}

The Electron capture rate of a nucleus $(Z, A)$ is contributed by individual capture rates $\lambda_{i f}$ from one of the initial states $i$ to all possible final states $f$ [9-12]. Considering the background magnetic field, the integrand in phase space of the capture rate reads

$$
f_{i f}=\frac{e B}{4} \sum_{n=0}^{n_{\max }}\left(2-\delta_{n 0}\right) \int_{\omega_{l}^{\prime}}^{\infty} d \omega_{B} \frac{\omega_{B}}{p_{B}}\left(Q_{i f}+\omega_{B}\right)^{2} F\left(Z, \omega_{B}\right) S_{e}\left(\omega_{B}, \mu_{B}, T\right),
$$

where the electron momentum transverse to the field direction is quantized into separate Landau levels, the maximum occupied level $n_{\max }$ is determined by the Fermi energy $E_{F}$ of the electrons (positrons). The electron energy $\omega_{B}$ is given by $\omega_{B}^{2}=p^{2}+m_{e}^{2}+2 e B . \mu_{B}$ denotes the chemical potential within the background magnetic field. The integration limits of Eq. 1 are set by the reaction threshold $\omega_{l}^{\prime}$. $Q_{i f}$ is the electron capture transition energy, determined from the nuclear masses. $F(Z, \omega)$ is the Fermi function which corrects the distortion of the electron wave function [13]. Inside the realistic astrophysical plasma, the interaction rates are varied from the vacuum case due to the many-body effect inside the plasma such as the Coulomb screening correction. The screening could mainly change: (1). the threshold energy for the capture is varied by the amount $\Delta Q_{c}=\mu_{c}(Z-1)-\mu_{c}(Z)$ [14], where $\mu_{c}$ is the Coulomb chemical potential [15]; (2). The energy of the captured electron $\omega$ is reduced by the screening potential $\Delta V(B)$, which is obtained under the relativistic degenerate condition $[6,7]$ (see Ref. [8] for more details).

In Fig. 1, we compare $f_{i j}$ under different density $\left(\rho Y_{e}\right)$ and field strength. For an extremely strong magnetic field, $E_{F}$ decreases monotonically as a function of $B$, only the Lowest Landau Level (LLL) is occupied, and the value of $f_{i j}$ shows the same trend since the Fermi energy determines the maximum energy of electrons. With decreasing magnetic field strength, the new Landau level pops up to cancel the extra energy carried by the magnetic field. Therefore, $f_{i j}$ decreases firstly, and then increases since the cancelling effect decreases monotonically with decrease of the magnetic field. Until the next Landau level pops up, such a pattern will be repeated. The left panel of Fig. 1 shows the ratio between screened and unscreened $f_{i j}$ : the screening could suppress $f_{i j}$ for about $20 \%$ under high density and strong magnetic field case (the right bottom part), while for a weak magnetic field, screening could reduce $f_{i j}$ only for few percent. This is consistent with the previous study [13] where only the field-free screening is taken into account. On the right panel, we compare the ratio of $f_{i j}$ between the scenarios with and without magnetic field. For every $\left(\rho Y_{e}, B\right)$ combination that corresponds to a popped-up new Landau level, $f_{i j}$ value could be enlarged for 1.5 times due to the larger value of $E_{F}$. Once the field strength becomes lower than $10^{14} \mathrm{G}$, it becomes the same value as the $B=0$ scenario. Since $\lambda_{i j} \propto f_{i j}$, such impact on the phase space should leave the imprints in the final electron capture rates.

\section{Electron Capture Rates of Iron Group Nuclei}

The importance of the shell-model rates in the pre-SN stage has been investigated previously $[2,3]$. It was shown that weak interaction rates significantly affect the central electron fraction $Y_{e}$ at the onset of the core-collapse, which further affect the nucleosynthetic path. The detailed analysis of $Y_{e}$ change after Oxygen burning also find that the critical flow during the final stages of stellar evolution is much closer to the valley of stability, and several essential electron capturing nuclei (e.g., ${ }^{54,56,58} \mathrm{Fe},{ }^{55} \mathrm{Mn},{ }^{53} \mathrm{Cr}$ ) are identified [2, 3]. Inspired by the previous studies, we first focus on the rate of electron capture on ${ }^{54} \mathrm{Fe}$. We calculate the reaction rates in different sets of stellar conditions (i.e., the combination of density, temperature 

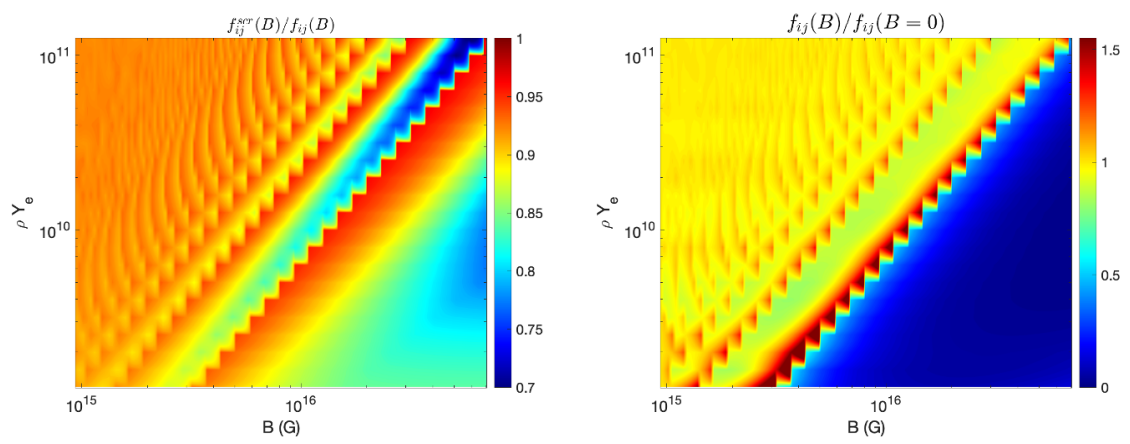

Figure 1. The left panel: The ratio between screened and unscreened $f_{i j}$. For the field-free situation, only a few percent deviations of $f_{i j}$ could be found (i.e., left-upper region of this panel). For the high field and relatively low-density case $\left(B>10^{16} \mathrm{G}\right.$ and $\left.\rho Y_{e} \sim 10^{9}\right)$, the screening effect could suppress $f_{i j}$ about $20 \%$. The right panel: The $f_{i j}$ in a background magnetic field compared with a field-free case. $f_{i j}$ could be enhanced about 1.5 times when the field strength just corresponds to a new popped-up Landau level. For the extremely high field case, only the LLL is allowed for the electrons to be occupied in, then $f_{i j}$ is drastically reduced (i.e., left-upper region of this panel). The calculation here sets $T=10 \mathrm{GK}$. This figure is taken from [8].

and $Y_{e}$ ). Those combinations are appropriate for the inner core of a collapsing $15 \mathrm{M}_{\odot}$ star, the profile of which is taken from Ref. [13]. Fig. 2 shows the reaction rate of ${ }^{54} \mathrm{Fe}\left(e^{-}, v_{e}\right)^{54} \mathrm{Mn}$

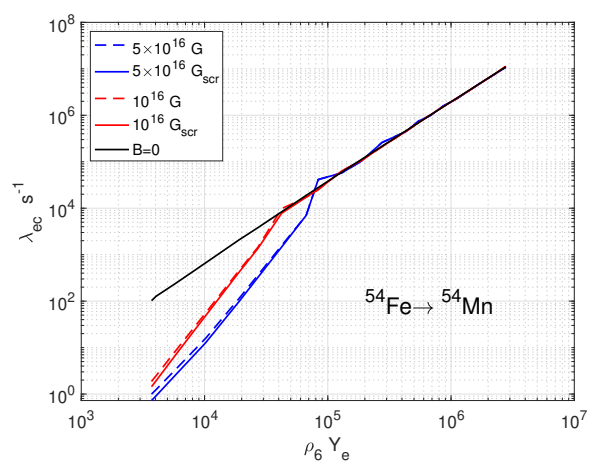

Figure 2. The reaction rate of ${ }^{54} \mathrm{Fe}\left(e^{-}, v_{e}\right)^{54} \mathrm{Mn}$ as a function of $\rho Y_{e}$. Blue lines correspond to a $10^{15} \mathrm{G}$ magnetic field. Red lines correspond to a $10^{16} \mathrm{G}$ magnetic field, and black lines are the field-free case. The screening corrections are also included as solid lines. This figure is taken from [8].

as functions of $\rho Y_{e}$. All the rates are converged to the same value for electron chemical potentials $\mu_{e}$ larger than about $25 \mathrm{MeV}$, while at lower $\mu_{e}$ values, the capture rates are more sensitive to the magnetic field strengths. The screening could suppress the electron-capture rates as expected, and the suppression is stronger for the lower $\mu_{e}$ value. It is also worth mentioning that, due to the magnetic field, $\mu_{e}$ is also a function of $B$, as discussed. Therefore, for the same combination of $\left(\rho, Y_{e}\right), \mu_{e}$ decreased as the increasing field strength, the electron capture rate could be reduced by almost two orders of magnitude for the low density and high field region. In high-density region, the field strength that allowed only the LLL occupation is also high, therefore $B<B_{\text {crit }}$ makes the indistinguishable trend of the reaction rates in 
high-density region. The electron capture process could reduce the electron number density and the neutrino emission from electron capture could carry away the energy and entropy of the core. Although the dominant composition during the collapse phase of the core is protons and neutrons, it has been shown that the heavy nuclei play an equally important role for the $Y_{e}$ evolution during the collapse [16]. Therefore, it is expected that such an influence of the magnetic field on the iron group nuclei could significantly impact the collapse and bouncing phase. It is worth extending to the sophisticated MHD-Jet SNe calculation.

\section{Conclusion}

In this work, the electron capture rates of iron group nuclei are re-evaluated for the strongly magnetized degenerate plasma. The degeneracy of the plasma makes the Thomas-Fermi approximation invalid. A relativistic generalization of the Hartree self-consistent field method is applied to calculate the strong screening potential. The screening potential could reduce the electron capture rate more significantly than the field-free situation. Moreover, the strong field strength only allows the lowest Landau level occupation. Therefore, the weak interaction rate is dramatically suppressed. A profile from a $15 \mathrm{M}_{\odot}$ core collapsing SN progenitor model is applied to calculate the electron-capture rates of ${ }^{54} \mathrm{Fe}$. It is noticed that two orders of magnitude reduction is found between the rates for plasma with density $\rho \sim 10^{9} \sim 10^{11} \mathrm{~g} \mathrm{~cm}^{-3}$ and magnetic field $B>10^{14} \mathrm{G}$. These impacts have potential significance since electron capture on iron group nuclei is the leading reaction that reduces $Y_{e}$, which finally could affect the $r$-process path and also SNe explosion models. Then, such results should be applied to the collapse and bouncing phases of MHD-Jet SNe calculation.

Y. L. is supported by JSPS KAKENHI Grant No. 19J22167. M. K. is supported by NSFC Research Fund for International Young Scientists (11850410441). T. K. is supported in part by Grants-in-Aid for Scientific Research of JSPS (17K05457 and 20K03958).

\section{References}

[1] G. C. McLaughlin, G. M. Fuller and J. R. Wilson, Astrophys. J. 472, 440 (1996).

[2] A. Heger, K. Langanke, G. Martínez-Pinedo and S. E. Woosley, Phys. Rev. Lett. 86, 1678 (2001).

[3] K. Langanke and G. Martínez-Pinedo, Rev. Mod. Phys. 75, 819 (2003).

[4] N. Nishimura, T. Takiwaki and F. -K. Thielemann, Astrophys. J. 810, 109 (2015).

[5] V. M. Kaspi and A. M. Beloborodov, Annu. Rev. Astron. Astrophys. 55, 261 (2017).

[6] A. E. Delsante and N. E. Frankel, Phys. Lett. A 67, 279 (1978).

[7] A. E. Delsante and N. E. Frankel, Ann. Phys. 125, 135 (1980).

[8] Y. Luo, Ph.D. thesis (The University of Tokyo, 2021); Y. Luo, M. Kusakabe, T. Kajino, M. A. Famiano and A. B. Balantekin, in preparation.

[9] G. M. Fuller, W. A. Fowler and M. J. Newman, Astrophys. J. Suppl. Ser. 42, 447 (1980).

[10] G. M. Fuller, W. A. Fowler and M. J. Newman, Astrophys. J. 252, 715 (1982).

[11] K. Langanke and G. Martínez-Pinedo, At. Data Nucl. Data Tables 79, 1 (2001).

[12] J. Pruet and G. M. Fuller, Astrophys. J. Suppl. 149, 189 (2003).

[13] A. Juodagalvis, K. Langanke, W. R. Hix, G. Martínez-Pinedo and J. M. Sampaio, Nucl. Phys. A 848, 454 (2010).

[14] R. G. Couch and G. L. Loumos, Astrophys. J. 194, 385 (1974).

[15] D. A. Shalybkov and D. G. Yakovlev, Astrophys. 27, 562 (1987).

[16] J. M. Sampaio, K. Langanke, G. Martínez-Pinedo, E. Kolbe and D. J. Dean, Nucl. Phys. A 718, 440 (2003). 